\title{
Philosophiques
}

\section{Le poète, le pédagogue et le républicain : Ginguené au service de l'instruction publique}

\section{Daniel Teysseire}

Volume 22, numéro 1, printemps 1995

URI : https://id.erudit.org/iderudit/027313ar

DOI : https://doi.org/10.7202/027313ar

Aller au sommaire du numéro

Éditeur(s)

Société de philosophie du Québec

ISSN

0316-2923 (imprimé)

1492-1391 (numérique)

Découvrir la revue

Citer cet article

Teysseire, D. (1995). Le poète, le pédagogue et le républicain : Ginguené au service de l'instruction publique. Philosophiques, 22(1), 117-135.

https://doi.org/10.7202/027313ar d'utilisation que vous pouvez consulter en ligne.

https://apropos.erudit.org/fr/usagers/politique-dutilisation/ 


\title{
LE POÈIE, IE PÉDAGOGUE ET LE RÉPUBLLCAN :

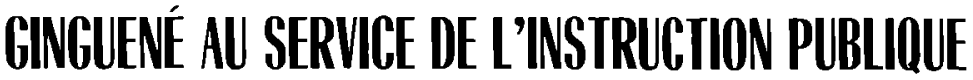

\author{
par \\ Daniel Ieysseire
}

Par et avec l'instauration et l'installation du Directoire, les Conventionnels et, plus généralement, les Révolutionnaires considèrent que la France est passée d'un « gouvernement révolutionnaire » (Décret du ig Vendémiaire an 2/ı Octobre 1793) provisoire de fondation de la République à un gouvernement constitutionnel stable de maintien de Celle-ci ${ }^{\mathrm{I}}$. Il s'agit donc désormais d'assurer ce maintien, et contre ses ennemis extérieurs, par une politique étrangère dynamique de présence armée - on continue donc la guerre, par ailleurs excellent adjuvant du trésor -, et dans la population de la France elle-même, par l'inculcation nécessaire des valeurs républicaines dans l'esprit des citoyens. La République sera pédagogue et pédagogique ou ne sera pas! Nest-ce pas d'ailleurs inscrit dans la Constitution de l'an 3, qui lie très explicitement citoyenneté et instruction en stipulant, à l' article 16 de son titre II, « État politique des citoyens »: « Les jeunes gens ne peuvent être inscrits sur le registre civique, s'ils ne prouvent qu'ils savent lire et écrire, et exercer une profession mécanique ». Et l'ajout qui dit que « cet article n'aura d'exécution qu'à compter de l'an 12 de la République » ne peut que pousser au développement de cette instruction publique républicaine. D'oủ tout un titre - le X - de la Constitution de l'an 3, consacré à l'« Instruction publique », avec six articles dont le zor si important pour l'inculcation de l'amour de la République, puisqu'il stipule qu'« il sera établi des fêtes nationales, pour

I. Suivant une distinction faite laconiquement par Robespierre dans son Rapport de Noël 1793 ( 5 Nivose an 2) sur Les principes du Gowvemement révolutionnaire fait à la Convention au nom du Comité de Salut Public (Robespierre, CEuvres, Tome $\mathrm{x}$ Discours, Paris, Presses universitaires de France, 1967, p. 273-282) : « Le but du gouvernement constitutionnel est de conserver la République; celui du gouvernement révolutionnaire est de la fonder » (p. 274). Il va sans dire et encore mieux en le disant que gouvernement n'a pas le sens restreint qui est le nôtre, mais celui, plus ample, de système politico-institutionnel ou forme d'Etat; ne parle-t-on pas en effet de «gouvernement monarchique»? 
entretenir la fraternité entre les citoyens et les attacher à la Constitution, à la patrie et aux lois $\rangle^{2}$.

Ces rappels d'histoire politique de la Révolution sont faits pour signifier que, même si nous considérions que Ginguené n'est pas un strict tenant du projet Idéologique, avec ses trois composantes fondamentales que sont le républicanisme progressiste, l'hygiénisme eugénique et le souci pédagogique ${ }^{3}$, nous devrions quand même et toujours le voir animé dudit souci pédagogique, tout simplement en tant qu'authentique révolutionnaire républicain. Mais alors se pose la question de l'inscription dans le réel de ce souci, de la traduction concrète de cette volonté pédagogique. Autrement dit : ces intellectuels en Révolution que sont les Idéologues ou leurs très proches, que font-ils très concrètement, quand ils ont le pouvoir effectif d'inculquer des valeurs par le biais de la transmission de connaissances? Importante et grave question, puisqu'il s'agit de rien moins que du rôle de l'intellectuel ou du savant en politique, de son pouvoir d'intervention dans le champ du politique, comme l'on dit maintenant, c'est-à-dire sur les finalités de la société. Comme on le voit, l'analyse de la micro-situation que je vais présenter s'inscrit dans la lignée des travaux du regretté Jacques Léonard ${ }^{4}$. (Que cette rapide mention soit prise comme un hommage profond au grand universitaire rennais !) sur la constitution du pouvoir scientifique et intellectuel, pour laquelle la Révolution est un moment fondamental, pour ne pas dire le moment fondateur.

Pour répondre à la question posée, nous allons examiner la situation donnée par et dans un corpus d'archives : celui des manuscrits se rapportant à l'action de Ginguené à la tête de l'administration de l'Instruction Publique, qui se trouvent dans le tome zo du fonds Parent de Rosan déposé à la bibliothèque municipale du XVI arrondissement de Paris. Le lecteur trouvera en annexe la liste des 16 pièces constitutives de ce corpus, classées par ordre chronologique

2. Toutes mes citations de la Constitution de l'an 3 sont tirées de l'ouvrage : Les Constitutions de la France depuis 1789, présentation par Jacques Godechot, Paris, Garnier-Flammarion, 1970, p. IOI-I4I.

3. Sur ce que j'appelle le projet Idéologique (= des Idéologues), ce sont plusieurs de mes articles qu'il faudrait citer ici; je me contenterai du plus récent : « La Fraternité, ciment de la République, chez les Idéologues Volney et Cabanis », dans La Fraternité, ciment de la République. Actes du 4 symposium humaniste international de Mulhouse, janvier 1992, Mulhouse, sous-presse.

4. Je pense, bien sûr, à La Médecine entre les savoirs et les pouvoirs. Histoire intellectuelle et politique de la médecine française au XIX $X^{\mathrm{e}}$ siècle, Paris, Aubier-Montaigne, 198I, $386 \mathrm{p}$. Toujours dans cette même ligne de recherche, citons également les travaux de Nicole et Jean Dhombres, et particulièrement leur Sciences et savants en France: naissance d'im pouvoir 1793 - 1824, Paris, Payot, 1989, 960 p. 
et intitulées par les premières lignes du document - ce qui,je l'espère, facilitera les recherches à venir, l'inventaire par ordre de documents du catalogue des manuscrits des bibliothèques n'étant pas un modèle de rigueur. De ces i6 pièces manuscrites, $\mathbf{g}$ concernent la situation dont il s'agit de faire l'analyse.

\section{Le problème politico-pédagogique à traiter}

Tout commence par la pièce ou document $n^{n} \mathbf{6}$, c'est-à-dire par la lettre de François de Neufchâteau, ex-Directeur fructidorien redevenu Ministre de l'Intérieur en Mai I798, demandant à l'ambassadeur rappelé de Turin, et donc disponible, de figurer « parmi les noms justement honorês des citoyens » ( $f^{\circ}{ }^{15} 8$ ) dont il a cru devoir composer le Conseil d'Instruction Publique dont il veut s'entourer. Réponse de notre Ginguené en date du 25 Brumaire an 7/Jeudi 15 Novembre 1798 :

[texte I] « C.en M.tre [Citoyen Ministre], Je sentois le besoin et le désir du repos: mes dernieres épreuves et l'état chancelant de ma santé en justifioient assez le projet; mais vous avez dispose de moi : vous me présentez l'espérance d'être utile : vous m'associez à des hommes dont il est honorable de partager les travaux et dont plusieurs sont mes amis; enfin vous me rapprochez, et vous choisissez pour vous occuper de moi l'instant de ce qu'on appelle vulgairement une disgrâce. Je se sais point résister à tant d'amorces, et j'accepte avec sensibilité ce que votre amitié m'a si noblement offert. Je m'estimerai très heureux si ce qui me reste de forces peut contribuer à l'amélioration des mœurs, à la propagation des principes republicains, à la prospérité de votre administration et à l'affermissement de la République. / ». (Doc. $7, \mathrm{f}^{\mathrm{i}} \mathrm{i} 6 \mathrm{0}$ recto) [= Texte intégrall.

C'est clair : ce n'est pas un fonctionnaire qui écrit à son Ministre; ce sont deux amis et deux frères en républicanisme qui correspondent et se rendent mutuellement service, en ne perdant pas de vue, bien sûr, l'intérêt supérieur de la République; à ce moment, rien moins que son ancrage dans la population. Bien avant la date, c'est déjà la République et des camarades et des professeurs.

Quasi-exactement cinq mois après, le même Ministre se tourne vers son ami, afin de lui demander d'examiner pour avis le résultat du travail confié au citoyen Parny « relativement au choix des meilleures poësies françaises depuis Marot jusqu'à nos jours, pour l'usage des Écoles nationales » (Document n' $\mathbf{g}$ en

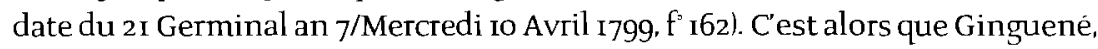
érigé ainsi en expert politico-pédagogique, se tourne vers ses collègues du Conseil d'Instruction Publique pour leur demander leur avis :

[texte 2] « Le but du Ministre a été de mettre entre Les mains des Jeunes gens un choix des meilleures poësies françoises, depuis Marot jusqu'à nos jours, dégagé non seulement de ce que Les meilleures poëtes offrent quelquefois de médiocre, mais de toutes Les expressions qui portent l'empreinte d'un régime aboli et d'un ordre social qui n'existe plus. Ce but est assurement très louable; mais l'extension donnée à ce recueil rend peutetre difficile de l'atteindre sans de graves inconveniens ». (Document n" I o non daté, f' I66). 
Ainsi, nous nous trouvons en face d'une configuration que nous connaissons bien; celle de la dialectique pouvoir politique-experts ou conseillers scientifiques ou techniques, le premier choisissant les seconds (ou simplement leur président), proches de lui idéologiquement - c'est le cas de le dire! -, mais cependant autonomes par leur compétence technique qui leur permet d'émettre un avis présenté alors comme une vérité non-partisane, mais, à l'évidence, idéologiquement marquée.

Synthétisons le problème qu'a à résoudre Ginguené. D'un côté. le ministre veut faire éditer un recueil de morceaux choisis de poésie française à l'usage des écoliers de la République; de l'autre, il en a confié la réalisation à un poète connu qui doit se charger de choisir et, si nécessaire, de modifier les textes pour que de jeunes oreilles, chastes et républicaines, ne soient pas choquées; question : une telle expurgation morale et politique est-elle compatible avec la finalité de connaissance de la poésie française, qui est celle d'un tel recueil. Question subsidiaire : quelle taille peut avoir celui-ci, car la poésje française de Marot à I798, ce n'est pas rien? Devant de telles difficultés, on comprend que notre auteur ait voulu prendre l'avis du Conseil:

[texte 3] « C.en M.tre Vous m'avez chargé d'examiner la première partie du travail du C.en Parny sur les meilleures poësies françoises depuis Marot jusqu'à nos jours, et vous m'avez fait un devoir de vous dire mon avis sur ce travail. J'y ai trouvé en le parcourant ce que Le nom de Son auteur fait attendre, un goût infini dans le choix, dans les suppressions et surtout dans les corrections et les substitutions qu'il a faites. mais après ce premier coup d'œil, il m'est venu une foule de réflexions et de doutes sur le fond même de l'entreprise, sur le but que vous vous êtes proposé en l'ordonnant et Sur le moyen choisi pour l'atteindre. Jai èté jusqu'à craindre que la malveillance ne s'exerçat et contre L'execution quelque parfaite qu'elle fut, et contre l'ordre même. l'intérêt que Je porte à tout ce qui vous regarde et l'espèce de responsabilité dont me chargeoit l'avis que votre confiance exigeoit de moi, tout m'a fait une loi de ne pas m'en rapporter à moi seul, de consulter sur cet objet important Le Conseil d'instruction publique où vous avez bien voulu me placer, et de vous transmettre aulieu de mon avis privé celui de ce Conseil, fait pour avoir un tout autre poids. J'ai pensé qu'en suivant cette marche /f 164 verso/ je ne vous en prouverois que davantage mon extréme désir de repondre à votre confiance et de seconder utilement votre zele pour la gloire des Lettres et celle de la Republique ». (Document n' 13 non daté, $f$ I64.)

Visiblement, la gloire des lettres et celle de la République peuvent être contradictoires ou, à tout le moins, divergentes. En effet, que sera un apprentissage de la poésie française qui se fera sur des textes qui ne seront pas originaux? Le moralisme et l'idéologie républicains y gagneront peut-être, mais au prix d'une renommée de la poésie française, qui, elle, sera bel et bien usurpée, puisqu'elle s'appliquera à des textes qui ne seront pas véritablement d'elle ou, plus exactement, des auteurs qui la constituent et ce, même si les 
remaniements sont parfaitement éxécutés. Avant de voir quelle va être la solution proposée par le Conseil, examinons sa composition.

\section{Les protagonistes de l'affaire}

Nous en connaisssons déjà trois : l'auteur qui nous occupe; le Ministre de l'Intérieur, François de Neufchâteau; et l'auteur sollicité pour composer le recueil de morceaux choisis : le poète Parny. Autrement dit, les trois personnages de mon titre : le pédagogue ou expert en pédagogie-Ginguené -, le politique républicain et le poète en charge de l'œuvre, chacun des trois, de plus, étant lui-même un peu les trois choses à la fois. Ce qui fait qu'ils s'entendent et se comprennent fort bien. Nont-ils pas d'ailleurs un autre point commun : leur appartenance à la loge maçonnique des « intellectuels », celle des Neuf Scurs $^{5}$ ? D'où, sans doute, cette manière qu'ils ont tous les trois de s'épauler entre eux. Le ministre a commandé le travail au ci-devant chevalier Parny, au demeurant « l'un des poètes français les plus distingués qui fermèrent un siècle tout philosophique » (Biographie Michaud, t. 33, [1823]l, p. 14.), cest-à-dire, en un mot, très populaire; l'expert nommé par le ministre, Ginguenè, ne peut que se féliciter de ce choix et du travail éxécuté (voir notre texte 3 cité cidessus). Quant au poète, il est prêt à faire ce que voudra bien proposer son ancien condisciple du Collège de Rennes:

[texte 4] «En Soumettant à vos Lumières autant qu'à votre autorité, Les representations de votre Conseil d'instruction publique, je me bornerai, Citoyen Ministre à vous prier de les prendre en considération, et d'inviter Le C.en Parny à reduire Son travail, au petit manuel poëtique que le conseil croit Suffisant pour l'Education Litteraire de la Jeunesse. vous ne pouvez en confier la Rédaction à des mains plus delicates et plus pures; et je ne dois pas vous cacher en finissant, pour donner un nouveau poids à L'opinion que je mets Sous vos yeux, que Le C.en Parny la partage, qu'il met à part tout ce qu'un travail long et penible a eu de fatiguant pour lui, et que si votre decision conforme à la délibération du Conseil, rend inutile tout ce travail, elle ne satisfera personne autant que celui qui l'a fait.

Salut et Fr ». (Document $\mathbf{n}$ I 3 non daté, f 165 recto, § 2 .)

On ne peut rêver plus grande solidarité entre le ministre commanditaire, l'éxécutant commandité, et l'expert intermediaire entre eux ! Mais qu'en est-il des autres experts sollicités par Ginguené, c'est-à-dire les autres membres du Conseil d'Instruction Publique?

5. Voir : Louis Amiable, Une loge maçonnique d' avant 1789 . La Loge des Neuf Saurs. Augmenté d'un commentaire et de notes critiques de Charles Porset, Paris, Edimaf, ig89. Les pages concernant nos trois premiers protagonistes sont les p. $304-308$ et $17 \mathrm{I}^{-1} \mathrm{I} 75$ du Commentaire critique, pour François de Neufchâteau et Parny, et les P. 321-323 et 186 , pour Ginguené. 
Le document I I, donné intégralement ci-dessous dans le point 3 (Le résultat), nous livre les neuf noms de ceux-ci. Le moins que l'on puisse dire de cette composition, c'est qu'elle est à la fois impressionnante et curieuse. En effet, quel est le président de cet organisme chargé de conseiller le ministre en matière de bonne éducation républicaine? Eh bien ! tout simplement, le vieux pourfendeur des philosophes, devenu ami des Théophilanthropes et admirateur de Bonaparte : Palissot ${ }^{6}$, toujours prêt à défendre Boileau ${ }^{7}$ dont le nom apparaît précisément dans le rapport final du Conseil (Doc. I I). Aux côtés de ce « vieil »- il a 69 ans - acteur des luttes intellectuelles des Lumières, on retrouve une bonne partie, et de l'équipe dirigeante, et de collaborateurs plus ou moins proches, de La Décade : Ginguené et Le Breton pour les premiers; Jacquemont, Domergue, Daunou, Tracy et Darcet pour les seconds, Lagrange étant un peu à part, mais jouissant de la sympathie de ladite Revue; autrement dit tout un réseau relationnel d'hommes constituant un milieu. Ici, bien sûr, ce sont des dizaines de pages du monument qu'est la thèse de Marc Régaldo ${ }^{8}$ qu'il faudrait citer; en particulier tout le chapitre V (p. 92I-I Ioz) consacré à « La Décade et l'Instruction publique ${ }^{9}$. Contentons-nous donc de quelques remarques. Constatons d'abord que la composition donnée par Marc Régaldo à la page $213^{10}$ du tome I de son ouvrage correspond à celle de notre document I I, sauf pour un nom, et un nom d'importance : celui de Destutt de Tracy, qui figure ici au lieu et place de celui de Garat là, très occupé par ses fonctions (Secrétaire puis Président) au Conseil des Anciens ${ }^{\text {II }}$. Que le père nominal de l'Idéologie fasse partie de ce Conseil contribue à tirer un peu plus celui-ci vers

6. Voir la rubrique d'Hervé Guenot consacrée à Palissot dans le Dictionnaire des journalistes (I680-178g) ss. la dir. de Jean Sgard. Supplément IV, Grenoble, Presses Universitaires de Grenoble, 1985, p. I69-188. Page i74, Hervè Guenot ècrit que, sous le Directoire, Palissot réussit à obtenir un appartement au Palais des Arts. «L'explication de cela est simple : le Conseil d'Instruction Publique se réunit le huit de chaque décade, à dix heures du matin [...] au Palais National des Sciences et des Arts, cour quarrée, coté du midi ». (Doc. 8, f $\mathrm{f}^{\mathrm{T}}$ т $\mathrm{T}$ ). Pourquoi son président n'aurait-il pas été logé dans le même palais, grace à l'intervention de son cher élève : François de Neufchâteau.

7. Ibid., p. 176.

8. Marc Régaldo, Un milieu intellectuel : La Décade philosophique (r794-1807) 5 tomes, Lille/ Paris, Atelier de reproduction des thèses/Librairie Champion, 1976, 1659 p. (Tomes I à III) + I4IO p. (Tomes IV et V).

9. Plus spécialement encore, les p. 929-930 consacrées au Conseil de l'Instruction Publique.

Io. Cest à la note I de cette page qu'est confirmée la grande « sympathie » de La Décade « pour le grand mathematicien » Lagrange.

II. Voir la notice consacrée à Garat dans : Lemay (Edna Hindie). Dictionnaire des Constituants 1789-I79I tome I, Paris, Universitas, 199I, p. 386-388. 
celle-là; à l'« Idéologiser » un peu plus, si je puis m'exprimer ainsi. Cabanis et Volney en feraient partie, ce serait un Conseil véritablement Idéologiste; tel qu'il est, c'est un Conseil « Décadiste » et, comme La Décade elle-mème, une «filiale » de la seconde classe de l'Institut National - celle des Sciences morales et Politiques - et, plus particulièrement, de sa section I - celle de l'analyse des sensations et des idées; à moins que ce soit l'inverse, à savoir que c'est La Décade qui est la maison-mère de toutes ces institutions, et donc du Conseil d'Instruction Publique. La deuxième remarque que l'on peut faire sur la composition de celui-ci concerne Domergue ${ }^{\mathrm{I} 2}$. Pourquoi ? Parce que ce maniaque de la fondation de sociétés linguistiques - à la date qui nous occupe il en est à sa quatrième ! - est l'homme de l'articulation du champ linguistique au patriotisme (au sens révolutionnaire) républicain. Autrement dit, il est l'expert par excellence en matière de langue - dans un sens très ètendu qui inclut la littérature : le «Prospectus »d'annonce de sa « Société des Amateurs de la langue Francoise » (179I) ne parlait-il pas déjà en effet des « lumières qui vont jaillir des débats littéraires » qui auront lieu dans cette Société ${ }^{13}$. C'est dire qu'il est le garant d'une bonne pédagogie républicaine de la littérature et ce, d'autant plus qu'il est, de tous les personnages cités de ce Conseil, celui qui a maintenu, sans problèmes majeurs durant les différentes phases de la Révolution, cette volonté de républicanisation de la langue et de la littérature. Qui retrouve-t-on d'ailleurs dans cette Société ? Robespierre. Palissot et... François de Neufchâteau ${ }^{\mathrm{I} 4}$ - déjà et encore!

Ce qui m'amène à ma dernière remarque sur les protagonistes du Recueil Neufchâteau-Parny. Ce groupe de personnes qui se connaissent, qui se fréquentent et qui travaillent ensemble depuis des années, avec l'outillage mental de la philosophie des Lumières tardives - la mouvance Idéologiste - et

12. Mieux connu maintenant grâce aux thèses de François Dougnac, F. U. Domergue, le Joumal de la Langue française et la néologie lexicale (1784-1795), Paris, Université de Paris III, I98I; Winfried Busse, F. U. Domergue (1745-1810). Die Rolle Domergues in der extemen Geschichte des französischen Sprache, Stuttgart, Universität Stuttgart, Ig8I.

On se reportera également au p. $69-80$ de Jacques Gulhaumou, La Langre politique et la Révolution française. De l'événement à la raison linguistique, Paris, MéridiensKlincksieck, ig89.

13. "Prospectus » cité in-extenso par Françoise Dougnac dans sa contribution à Winf ried Busse et Jürgen Trabant (dir.): Les Idéologues. Sémiotique, théories et politiques linguistiques pendant la Révolution française. Actes du Colloque de Berlin, octobre 1983 . Amsterdam/Philadelphie, John Benjamins, 1986, p. 312-316. Quelques lignes plus loin que celle de la formule citée, Domergue parle de « notre littérature let pas seulement "notre langue"l » comme d'une "aristocratie oppressive et decourageante $»$ (p. $315=$ p. 3 du « Prospectus $»)$.

I4. Ibid., p. 309 et 310. 
le choix politique révolutionnaire-républicain, ne constitue pas seulement un « milieu intellectuel », pour reprendre l'expression de Marc Régaldo, mais aussi et tout autant un groupe de pouvoir, l'un étant indissociable de l'autre. C'est bien la première fois dans notre histoire, me semble-t-il, que nous avons affaire à un pouvoir scientifique (Darcet et Lagrange ne sont pas les derniers venus) et intellectuel laïc constitué, et agissant en tant que tel par et au nom de la compétence experte. J'ajoute que l'existence de ce groupe atteste la continuité longue d'un certain personnel révolutionnaire - je ne dirai même pas par-delà, mais bien tout au long, des différentes phases de la Révolution. En tant que processus évolutif - à rapidité variable - celle-ci a eu besoin d'un substratum minimal de continuité; les intellectuels et savants et les idées des Lumières tardives l'ont constitué.

\section{Le résultat : la solution proposée par Cinguené et Ie Conseil d'Instruction Publique}

Ces hommes ayantà résoudre le problème de la mise en place d'un recueil pédagogique de poesie française, que vont-ils décider qui préserve à la fois la nécessaire inculcation des éléments fondamentaux du système républicain et la non moins nécessaire inculcation de la connaissance et du goût de ladite poésie, ces deux inculcations nécessaires pouvant être, comme on l'a vu, parfaitement divergentes ? La réponse est donnée par et dans notre document I I qui livre le résultat de la déliberation du Conseil d'Instruction Publique, dont je préfère donner l'intégralité du texte plutôt qu'une glose faite de citations en substance. Puisqu'à ma connaissance le texte est inédit, autant le publier tel quel. Il constitue ainsi l'essentiel de ce troisième point de mon analyse.

[texte 5] « Extrait du procès-verbal de la séance du i8 floréal an 7 de la Rép.que F.re, composée des citoyens Palissot, Darcet, Daunou, Domergue, Ginguené, Jacquemont, LeBreton, Tracy, Lagrange.

«Un membre a dit : Citoyens, Le Ministre de l'intérieur m'a chargé d'examiner le travail d'un de nos meilleurs Poëtes actuels Sur les ouvrages de ceux qui ont les premiers prêté à notre Langue les Charmes de la Poësie : ce travail doit être poussé plus loin, et s'étendre Jusqu'aux poëtes qui ont vécu de nos jours : il m'a paru de nature à ne pas me permettre de prendre Sur moi l'examen, sinon du détail et de l'exécution, au moins du principe Sur lequel il est fondé et de l'esprit Général dans lequel il est conçu. J'ai cru devoir consulter à cet égard le Conseil d'instruction publique, lui Soumettre mes réflexions, m’eclairer de ses lumières et m'appuyer de son autorité.

«Le but du Ministre a été de mettre entre les mains des jeunes Gens un Choix, des meilleures Poësies françoises depuis Marot jusqu'à nos jours. dégagé non seulement de ce que les meilleurs Poëtes offrent quelquefois de médiocre,/f 169 verso/ Mais de toutes les expressions qui portent l'empreinte d'un Régime aboli et d'un ordre Social qui n'existe plus. Ce but 
est assurément très louable; mais l'extension donnée à ce Recueil rend peut être difficile de l'atteindre sans de graves inconvéniens.

« Une notice de la vie de chacun des Poëtes doit précéder les meilleures pieces qui sont restées de lui. elles sont peu nombreuses, comme on doit le Penser, depuis Marot jusqu'à Malherbe; mais à compter de ce dernier qui fixa la langue Poëtique, chaque Poëte fournit d'avantage; on Sent enfin que Boileau, La fontaine, J.B. Rousseau et Voltaire doivent, à eux Seuls, remplir plusieurs volumes. ainsi la Collection entière en contiendra t'elle huit ou dix.

«Dans ce Système, on doit admettre toutes les Pièces dignes de la Réputation de leurs auteurs; mais les pièces les plus dignes de leur Réputation portent de fréquentes empreintes des opinions, des đignités, des Servitudes, et des préjugés de leur tems. or le Ministre a voulu qu'il n'y eut dans tout le Recueil aucune expression monarchique, aucune de libre. aucune qui fût relative à un Culte quelconque; et partout où il s'en trouvoit, dans les pièces les plus généralement connues et estimées, il a fallu pour remplir cette intention, que le Rédacteur du recueil retranchât, modifiât, otât un vers, quelquefois et $y$ en Substituât d'autres. Cest cette partie de son travail qui excite mes craintes, éveille mes doutes et me paroît mériter toute l'attention du Conseil. /f $170 /$ heureusement il a été confié à l'un de nos Poëtes le plus en état de le bien faire, de le faire avec goût et avec une espèce de religion littéraire. il n'a retranché qu'avec scrupule, et le plus souvent sa couleur se marie si bien avec celle des originaux qu'il répare, que, pour me servir d'un terme qui, pour la première fois peut être, peut passer avec justesse de la Peinture à la Poësie, il seroit difficile d'appercevoir les Repeints.

«[...] heureusement encor l'homme le plus capable de cette opération, devoit le mieux en Sentir les inconvéniens; aussi mettant à part ce qu'un travail long, pénible et fastidieux a eu de fatiguant pour lui, partage-t-il mon opinion sur les points délicats que je soumets au Conseil; et je ne dois pas vous dissimuler, Citoyens, que si votre decision est conforme à mes doutes, et rend nul par Conséquent tout ce travail, elle ne Satisfera personne autant que celui qui l'a fait.

«L... je n'entrerai point ici dans le détail des raisons pour et contre : elles Seront développées dans la discussion que je vous prie d'établir à ce Sujet. je vous demande la permission de poser ainsi les questions que je soumets à votre examen.

«I' Sans parler d'autres Poëtes moindres en renommée et en mérite, n'y a-til pas une espèce de violation, à mutiler, retoucher, prétendre refaire des vers de Marot, de Malherbe, et plus encor de la fontaine, de Boileau, de Rousseau et de Voltaire? N'est-ce pas en quelque sorte un Crime et un Sacrilége littéraires? le Commettre, et, disons le même avec le /f r ro verso/ Sentiment qui nous attache tous au Ministre de l'Intérieur, l'ordonner, n'est-ce pas S'exposer à des reproches graves?

« 2 Est-il nécessaire de mettre entre les mains de la jeunesse un recueil aussi etendu? ne suffirait-il pas de deux ou trois petits volumes pour lui donner l'idée et même le goût de notre Poësie? dans cet utile Compendium ne pourroit-on faire entrer ce que châque Poëte a de plus exquis? et n'y éviteroit-on pas facilement toutes ces expressions malsonnantes pour des oreilles Républicaines?

" 3 Enfin dans un Recueil ainsi réduit lorsqu'une piéce excellente et impossible à Supprimer renfermeroit quelques unes de ces expressions 
religieuses ou monarchiques, car pour les expressions libres, elles en seront entièrement bannies, une simple note, telles que l'on en met aux auteurs anciens pour l'explication des termes Mythologiques et historiques, ne Suffiroit-elle pas, au lieu d'une mutilation ou alterration quelconque du Texte?

«Telles sont, Citoyens, les questions intéressantes que je soumets à votre goût et à votre profonde sagesse. Jen écarte d'accessoires, qui pourront trouver place dans la discussion. ce ne sera point mon avis que je transmettrai au Ministre en répondant à la lettre qu'il a bien voulu m'écrire; ce sera celui du Conseil : il doit avoir un tout autre poids à ses yeux; et la marche que je Suis ne lui prouvera que d'avantage mon extrême desir de répondre à sa confiance et de Seconder utilement son zèle pour /f $17 \mathrm{I} / \mathrm{la}$ gloire des lettres et celle de la République

« Le Conseil arrête que les questions qui viennent d'être ainsi proposées par un de ses membres, feront dans sa prochaine séance, l'objet d'une Délibération particulière.

Suit, sans discontinuité dans le texte:

«Extrait du Procès Verbal de sa séance du Vingt huit floréal, an Sept, de la république française /Vendredi i 7 Mai I799/. Composee des Citoyens Palissot, Darcet, Daunou, Domergue, Ginguene, Jacquemont, Le Breton, Tracy, Lagrange.

«Dans la dernière séance, un Membre avoit soumis à la décision du Conseil plusieurs points de discussion relatifs au Projet qu'à le Ministre de l'intérieur, de faire Composer un Recueil Élémentaire des meilleurs morceaux des Poëtes françois depuis Marot jusqu'à nos jours; ce Recuei] devant comprendre un grand nombre de passages des Poètes qui ont Vécu sous la Monarchie, la question présentée au Conseil porte sur l'intention où est le Ministre, de faire changer ou supprimer les passages qui offriroient l'empreinte des préjugés ou des usages de ce tems, et particulièrement sur les inconvénients que semble offrir une semblable entreprise;

«I...I sur quoi le Conseil après avoir délibéré, Considérant qu'il ne peut y avoir de danger à laisser à la jeunesse Républicaine, le droit de s'instruire par la lecture de nos Poëtes, des Préjugés ou des habitudes existant sous l'ancienne forme de Gouvernement, comme elle a le droit de s'en instruire par l'étude de l'histoire; Considérant qu'il est même assez vraisemblable qu'à /f' 17 I verso/travers les prestiges de la Poësie ces usages Puérils ou Superstitieux se trouveront assez fidèllement retracés aux yeux des jeunes lecteurs, pour qu'ils puissent en saisir ou le Ridicule ou le danger; que peut être même les changements qu'on essayeroit d'y faire, les substitutions de mots, de vers, de phrases, en excitans la curiosité ou la malignité des lecteurs pourroient produire un effet tout contraire à celui qu'on s'en promet, et que d'ailleurs quelques soins quelque précaution qu'apportat à cette opération la main délicate qui en est chargée, de nouvelles variantes apposees ainsi aux Vers de Racine ou de Boileau, laisseroient toujours le Regret des morceaux de la $\mathbf{r}^{\text {ére }}$ version qu'un long usage a consacré; Considérant, d'un autre Coté, que, dans cette circonstance, le seul parti que puisse prendre le Conseil est d'autoriser celui de ses membres qui veut bien le consulter sur ce point à s'appuyer de son assentiment unanime pour obtenir du Ministre qu'il renonce à une idée dont le résultat ne répondroit probablement pas au vou bien reconnu de ce Magistrat pour la gloire de la République et celle des lettres, prend la Délibération suivante. 
« art. $\mathrm{I}^{\mathrm{er}}$.

Le Conseil autorise celui de ses membres qui l'a Consulté Sur les questions ci dessus relatées à faire connoître le résultat de sa présente délibération au Ministre de l'Intérieur, dans la conférence qu'il se propose d'avoir avec lui sur cet objet.

$\ll / f^{\mathrm{e}} 172 /$ art. $\mathbf{2}^{\mathrm{e}}$

«[... à cet effet ce membre recevra du Conseil expédition de la Délibération qui a été prise sur sa proposition.

«Pour Copie conforme Vincent Campenon».

L'intérêt de ce texte intégral à l'orthographe et à la ponctuation originales est double:

- concernant l'ensemble du dossier, il livre le point de vue complet de Ginguené, puisqu'il reprend exactement les termes de la pièce ro et annonce ceux de la pièce $\mathbf{3} 3$, dont l'inventaire figurant en annexe jointe ne donne que les titres. Le contenu de ces deux pièces peut donc être ainsi connu par le lecteur;

- pour ce qui est du fond du problème, il montre que le conseil d'Instruction Publique a concentré son attention de groupe d'experts sur la question de l'inculcation des références politiques et laïques (= a-religieuses) de la République, en sachant fort bien lier remaniements des textes, acquisition de connaissances poétiques et transgression des interdits. Si l'on modifie les textes, suggére le Conseil, pour empêcher les jeunes de connaître les réalités de l'Ancien-Régime, non seulement on ne leur fera pas découvrir et donc apprécier la poésie française originale, mais on risque à coup sûr de leur donner l'envie d'aller voir les textes originaux, parés ainsi de toutes les beautés qui ne manqueront pas alors de rejaillir sur le contenu politique et religieux desdits textes. Bénéfice net de cette manipulation des textes pour raison idéologique (au sens d'aujourd'huil : ni connaissance des textes poétiques, ni dégoût de l'Ancien Régime. Conclusion sage à tirer de tout cela : laissons les textes en leur état primitif et donc eventuellement descriptif d'une société et d'une religion passées. Le recueil de morceaux choisis projeté par le Ministre deviendra alors et un manuel de poésie et un manuel d'histoire. Quant à la moralité et à l'étendue d'un tel ouvrage, le Conseil ne semble pas avoir d'opinion, signifiant ainsi le caractère très politique de ce groupe d'experts, dont le remplaçant de François de Neufchâteau, Quinette, entérine la décision (Doc. 14.). Décidément, il n'est pas facile de faire du neuf avec du vieux ! 
Ce qui nous ramène à notre point de départ, c'est-à-dire à la mise en place du jeu dialectique entre le pouvoir intellectuel et scientifique, et le pouvoir politique. Que peut-on en dire à la lumière de l'analyse de la situation donnée par notre corpus?

- D'abord, que l'on pourrait penser naïvement que, pour que ce jeu existe, il faut que chacun des deux protagonistes de celui-ci existe également, chacun séparément. D'un côté, le pouvoir politique qui existe depuis longtemps - ô combien ! - même si la Révolution l'a fait changer de nature et de contenu; de l'autre, le pouvoir intellectuel et scientifique tout récent, du moins dans sa forme de pouvoir constitué en tant que tel et non plus dispersé entre des individualités magnifiées. Certes!

- L'ennui, c'est que le modeste exemple, la micro-situation que nous avons analysée montre que ce n'est pas aussi simple. Que voyonsnous en effet? Que le pouvoir politique républicain, c'est-à-dire - en nous souvenant de ce que nous disions en introduction à la suite de Robespierre - un pouvoir constitutionnel chargé de « conserver la République » suscite des institutions, des « pouvoirs constitués », comme l'on dit de nos jours. Ainsi ce Conseil d'Instruction Publique. La Révolution n'a pas besoin de savants en tant qu'individualites magnifiées, mais la République a grand besoin de savants constitués en corps qui lui indiquent l'efficacité utile. Sur notre exemple : quel bon manuel de poésie française pour l'enseignement républicain.

- On tient là l'explication du fait que le pouvoir intellectuel et scientifique est consubstantiellement lié au fonctionnement du pouvoir politique républicain. En effet, quelle est la finalité de celui-ci, sinon celle que n'ont cessé de poser les Lumières, à savoir la plus grande utilité commune possible. Si ni la naissance ni la grâce de Dieu ne légitiment ni ne soutiennent plus le pouvoir politique, qu'est-ce qui peut le faire ? Réponse : la participation à l'utilité commune, c'està-dire la compétence experte. Et voilà pourquoi et comment un ministre s'entoure d'un Conseil d'intellectuels et de savants qui lui pointeront du doigt l'efficacité de sa décision, au nom même de leur compétence d'experts.

Ensuite, le processus d'actions réciproques entre les deux instances peut avancer tout seul, facilité, bien sûr, par le fait que les hommes de chacune de celles-ci appartiennent tous au même milieu intellectuel et au même groupe de pouvoir, et sont donc tous des experts interchangeables. Le politique choisit dans son monde idéologique son groupe d'experts, les nomme; ceux-ci 
expertisent, et celui-là prend une décision qui n'est que l'entérinement de ladite expertise. La République des camarades, vous dis-je!

Faculté de Lettres et de Sciences Humaines

Université Paris XII - Val de Marne

Groupe de recherche en épistémologie et histoire de la médecine (G.R.E.H.M.)

\section{Annexe 1}

\section{Inventaire des 16 pièces du I. 20 du fonds Parent de Rosan, concernant l'action de Ginguené à la tête de l'administration de l'Instruction Publique (Ordre chronologique)}

- I. Paris, 27 Pluviose an 3/ Dimanche 15 Février I795 (f $235-238$ sans $237=6$ p. et demie.) :

«Chalmel ex-Secrétaire général de la Commission de l'instruction publique à Garat et Ginguené Commissaire et adjoint de la même Commission ».

- 2. Paris, rer. Ventose an 3/Jeudi ig Février 1795 (f 239 recto et verso) :

« J. L. Chalmel Secrétaire général de la Commission de l'instruction publique aux citoiens Garat et Ginguené membres de la même Commission $»$.

- 3. Paris, rer. Ventose/ Jeudi ig Février Isans autre précisionl ( $f^{\prime \prime 240) ~: ~}$

« Garat et Ginguenè à Chalmel ».

- 4. Sans date, mais, en bas du document ( $\mathrm{f}$ I54 verso), l'avis du Comité indique 18 Germinal an 3 / Mardi 7 Avril 1795 (f I53 $^{+}$I54) :

« Rapport au Comité d'instruction publique », signé « L'adjoint à la Commission exécutive de l'Instruction publique Ginguené ».

- 5. Paris, 3 Fructidor an 3 / Jeudi 20 Août I795 (f I5o recto et verso) :

« EXTRAIT DU PROCES-VERBAL DE LA CONVENTION NATIONALE, du

Deuxième jour de fructidor l'an troisième de la République française une et 
indivisible ». /Imprimé, sauf mentions du jour et du mois, au dessous du sceau de la Convention : l'œil de la vigilance./

«La Convention Nationale, sur le rapport de ses Comités d'Instruction publique et des finances, décrète : [...]»

- 6. Paris, 22 Brumaire an 7 /Lundi 12 Novembre 1798 (f $158+159$ vierge) :

« 5 Division. Bureau des Musées fr. /En-tête de la République : "Liberté." et "Egalité" encadrant le sceau de la "Répubuque FrançaisE"/ Le Ministre de l'Intérieur.

“Au Citoyen Ginguené." [...] »

- 7. Paris, 25 Brumaire an 7 / Jeudi 15 Novembre 1798 (f' i6o recto) :

«Au Ministre de l'Intérieur de

Paris 25 Brumaire an $7[$ [...]»

[= notre texte $\mathrm{I}$.

- 8. Paris, 29 Brumaire an 7 / Lundi ig Novembre 1798 (f" I6 r recto) :

« Le Secrétaire du conseil d'instruction publique, au Citoyen Ginguené, Membre dud. Conseil ».

- 9. Paris, 21 Germinal an 7 / Mercredi io Avril 1799 (f: $162+163$ vierge):

" $5^{\mathrm{e}}$ Division. Bureau du Secrétariat. /En-tête de la République : "Liberté." et "Égairté" encadrant le sceau de la "Répubuique Française"/ Le Ministre de l'Intérieur,

«Au Citoyen Ginguené, Membre de l'Institut et du conseil d'Instruction publique.[...]»

- Io. Sans date (f $f^{\circ} 166+I 67=4$ p. pleines):

«Citoyens,

«Le Ministre de l'Intérieur m'a chargé d'examiner Le travail d'un de nos meilleurs poëtes actuels Sur les ouvrages de ceux qui ont les premiers prété à notre Langue Les charmes de la poësie [...]»

[= pour partie, notre texte 2$]$

- I I. [Paris], I8 floréal an 7/Mardi 7 Mai 1799 (ff* 169 à I7I, avec en-tête du « Ministère de l'Intérifur, Conseil d'Instruction Publioue » précédée de « Liberté » et « Égalité » encadrant le sceau de la République : « Unité. Indivisibilité de la République FrançAise » [en majuscules]) :

«Extrait du procès-verbal de la séance du i8 floréal an 7 de la Rép.que F.re, composée des citoyens Palissot, Darcet, Daunou, Domergue, Ginguené, Jacquemont, LeBreton, Tracy, Lagrange. [...] »

[= notre texte 5. ]

- 12 : Paris, 8 Prairial an 7 /Lundi 27 Mai 1799 (f ${ }^{\circ} 68$ recto + I mot verso avec la formule de politesse) :

« Le Président du Conseil d'Instruction publique limprimél au Citoyen Ginguené, Membre du Conseil d'Instruction Publique [...] $\gg$

- I3 : Sans date (f $164+165$ recto seulement) : 
«C.en M.tre Vous m’avez chargé d'examiner la première partie du travail de C.en Parny sur les meilleures poësies françoises depuis Marot jusqu'à nos jours, et vous mavez fait un devoir de vous dire mon avis sur ce travail $[\ldots] \gg$.

[= Pour partie, nos textes 3 et 4.$]$

- 14 : Paris, le 20 Messidor an 7/Lundi 8 Juillet 1799 « de la République française, une et indivisible ». ( $\mathrm{ff}^{\circ} 173$ et 174 [vierge]) :

«Le Ministre de l'Intérieur, au C.en Ginguené, Membre de l'Institut National. [...]»

- I5 : Paris, le 27 floréal an 8/Samedi i7 Mai I80o (f ${ }^{-}$i75):

« J. A. Chaptal, Conseiller d'Etat, et Membre de l'Institut National, au Citoyen Palissot, Président du Conseil d'instruction publique. (.... »

- 16 : [Parisl, le 9 P.al an 8/Jeudi 29 Mai 1800 (ff' 176 et 177 lviergel) :

"Le Secretaire ["Président" ayant été rayél du Conseil d'Instruction publique Au citoyen, Ginguené, Membre de ce Conseil. [...]»

\section{Annexe 2}

\section{La tuberculose pulmonaire de Ginguené décrite par Moreau de la Sarthe}

ENCYCLOPÉdIE MÉTHodIQUE, « Dictionnaire de Médecine », Tome II, Paris, Veuve Agasse, 1824 .

p. 742 D. 1. $4-763$ D. 1. 60 : « PHTHISIE, s.f. (Pathol. génér. \& spéciale) ». (Moreau de la Sarthe).

p. 755 D. $1.34-76$ I D. 1. 4 I : « PHTHISIE TUBERCULEUSE ».

p. 759 D. I. $24-76$ I G. l. 12 :

«|...]

« Le troisième période est caractérisé par un progrès sensible, effrayant, dans des phénomènes particuliers de la maladie $\&$ dans les phénomènes généraux qui les accompagnent, telles que la fièvre dont nous venons de parler, l'insomnie, l'alteration des fonctions nutritives. Quelquefois cependant, ces symptômes généraux ne sont pas dans un rapport exact avec la disposition tuberculeuse, $\varepsilon$ font périr les malades d'une manière prématurée, et même avant d'avoir rendu des crachats jaunes $\varepsilon$ opaques : ce qui suppose toujours un grand nombre de tubercules. Dans d'autres circonstances, les phénomènes du râle muqueux, de la pectoriloquie[*| (I), \& l'expectoration la plus défavorable, se montrent, bien que l'embonpoint $\varepsilon$ un état de santé supportable paroissent encore exister. Il n'est pas même sans exemple, que certains malades qui n'avoient qu'un petit nombre de tubercules, aient parcourus tous les périodes du développement, du ramollissement, d'évacuation de matière tuberculeuse, en paroissant 
tourmentés par une toux nerveuse ou par une affection catarrhale l*l. La phthisie ou consomption n'existe pas, il faut l'avouer, dans ces cas fort rares $\varepsilon$ très-heureux, puisqu'elle résulte d'un progrès simultané dans les symptômes particuliers de l'état tuberculeux du poumon, $E$ dans l'influence désastreuse de cet etat, sur l'ensemble de l'organisme, tels que l'amaigrissement, l'insomnie, $6 c$.

« Ip. $760 \mathrm{Col}$ G. I Les symptômes qui amènent cette désastreuse influence $E$ tous les phénomènes qui se rapportent d'une manière directe à la disposition tuberculeuse, se prononcent de plus en plus dans le troisième période. L'embonpoint $E$ une apparence de santé qui s'étoient conservés jusqu'à cette èpoque, diminuent sensiblement : les forces se perdent, le déperissement augmente; la fièvre est continue, $\varepsilon$ les redoublemens sont annoncés de la manière la plus pénible, par une exacerbation dans tous les symptômes graves, telles que la toux, l'oppression, l'insomnie, Ec.

« D'autres symptômes qui appartiennent plus particulièrement à la troisième période, ne tardent pas à se montrer : telles sont l'œedématie l*l des extrémités inférieures, les sueurs colliquatives [*I $\varepsilon$ la diarrhée, que l'on attribue à une entérite consécutive $\varepsilon$ trop souvent dépendante d'une invasion de l'intestin, par la dégénérescence tuberculeuse. À cette époque de la maladie, les progrès du marasme sont effrayans, $\&$ si les malades ne périssent pas d'une manière prématurée $\varepsilon$ en succombant à des symptômes nerveux qui se joignent si souvent à toutes les maladies organiques, ils périssent dans un état de consomption $\&$ de destruction progressive, dont nulle autre maladie peut-être, ne présente l'exemple. Ce dépérissement, lors même qu'il ne seroit pas accompagné des autres symptômes généraux de la phthisie tuberculeuse, que l'on seroit parvenu à suspendre, suffiroit pour dissiper tous les doutes ou toutes les esperances que l'on pourroit conserver dans cette circonstance, $\varepsilon$ avec apparence d'amélioration.

« $\Rightarrow>$ Un des hommes de lettres les plus distingués des temps modernes, le savant auteur de l'Histoire littéraire de l'Italie, m'a offert, il y a environ seize ans, un exemple bien pénible de cette triste vérité. Je commençai à lui donner des soins à l'époque très avancée d'une affection de poitrine, dont il faisoit remonter l'origine jusqu'aux premières années de sa jeunesse, $\&$ pour laquelle il fut soigné pendant long-temps par un médecin qui ne s'étoit jamais fait une idée exacte de sa maladie $G$ qui l'avoit attribuée tantôt à la goutte, tantôt à un rhumatisme, tantôt à un embarras gastrique ou à une disposition érysipélateusel*]; à tout enfin, excepté à sa cause véritable $\&$ prochaine fl'état tuberculeux du poumon).

« Il me fut impossible, à la vérité, de reconnoitre d'une manière positive cette cause prochaine $\varepsilon$ ce genre de lésion qui ne pouvoit laisser aucune espérance, n'ayant encore eu, à cette époque, aucune idée de l'auscultation médiate $\varepsilon$ de l'usage du stéthoscope. Je les soupçonnai en donnant toute l'attention que je devois à une suite d'observations sur la complexion du malade, la marche de sa maladie, l'enchaînement de ses symptômes, l'augmentation progressive $E$ journalière du dépérissement, même à une époque où je parvins, par [col. $D$. I des médications convenables, à rétablir le sommeil $\mathcal{E}$ les fonctions digestives, tout en faisant cesser les sueurs nocturnes, la diarrhée, l'abondance de l'expectoration.

"Mr. G** passa une année à la campagne avec une apparence de convalescence, sur laquelle j'étois le seul de ses amis qui ne pût se faire illusion, averti de son danger, $\&$ par l'oppression qui devenoit chaque jour plus grande, $\Xi$ par la maigreur quí se montrait aux yeux les moins prévenus, 
avec tous les caractères du marasme. Tout-ä-coup, $E$ sans le retour des symptômes que j'avois combattus, les forces se perdirent entièrement chez Mr. G** : il lui sembla qu'un poids énorme l'accabloit $\&$ l'empêchoit de marcher, jusqu'à ce moment, il ne s'étoit pas cru malade, \& frappe de ce changement, il me fit appeler à sa campagne, où il venoit de passer dans un doux loisir, les momens les plus heureux de sa vie. Dès qu'il m'aperçut, il m'annonça que tout avoit bien changé depuis notre dernière entrevue, $\varepsilon$ qu'il avoit passé d'une situation de valétudinaire assez douce, dans un état bien déterminé de maladie. Nous le ramenâmes à Paris le lendemain, avec beaucoup de peine, $\&$ deux jours après il avoit cessé d'exister : toujours fatigué de plus en plus de cette sensation d'un poids qui l'accabloit même dans la position horizontale, \& qui lui paroissoit être la seule cause de son oppression. Quelques jours avant de mourir, il y eut des crachats d'apparence purulente $\varepsilon$ mêlés à des matières noirâtres, $E$ donnant à penser que la mélanose[*l s'étoit jointe sans doute à la dégénérescence tuberculeuse.

«Mr. G** vécut pendant plus de quarante ans sous l'influence de l'état morbide, dont il avoit ressenti les premiers symptômes dès l'âge de quinze à dix-huit ans, époque où il eut plusieurs hèmoptysies[*] consécutives : des soins entendus, plus éclairés, un genre de vie moins orageux que le sien, dans la période de cinquante à soixante ans, auroient probablement contribué à le soulager, en s'opposant à de nouveaux ramollissemens de tubercules $\varepsilon$ à la cicatrisation des cavernes tuberculeuses qui s'étoient formées probablement dans le poumon pendant long-temps, sans altérer sensiblement sa santé, qui ne fut complètement engagée, que lorsque le mal étant porté à son comble, il devint un obstacle insurmontable à toute nutrition. Il est important de dire, en terminant cette observation, que le fond de la complexion de Mr. G** étoit sensiblement remarquable par une dégénérescence rachitique qui ne s'ètoit pas développée; ce qui lui donnoit quelqu'analogie, sous ce rapport, avec l'un de ses plus illustres compatriotes, chez lequel cette disposition est annoncée évidemment $E$ par la prédominance du cerveau sur les autres organes, \& par le développernent extraordinaire des facultés mentales, qui coincide avec cet excès de volume, dans les masses cérébrales.

« $[p .76 \mathrm{I} \mathrm{Col}$ G. I Les archives de la médecine pratique présentent plusieurs exemples de ces développemens prolongés, souvent consécutifs $\mathcal{G}$ comme interrompus, de la phthisie tuberculeuse. Dans ces circonstances, la maladie présente des alternatives de convalescence imparfaite $\varepsilon$ de rechute incomplète, que l'on attribue souvent, sans raison, à différentes causes occasionnelles. La marche de la phthisie peut en outre se trouver embarrassée $\&$ compliquée par plusieurs autres maladies qui la font perdre de vue, $\&$ qui jettent souvent dans des méprises graves sur son traitement. «[... $\mid »$.

D'après ces lignes de Moreau de la Sarthe, la chronologie de l'évolution de la tuberculose pulmonaire dont est mort Ginguené pourrait s'établir de la manière suivante:

- une prédisposition native due à une constitution rachitique; 
- une tendance aux affections pulmonaires dès la jeunesse, avec crachements de sang dès $15^{-18}$ ans, soit entre 1763 et 1766 ;

- 40 ans d'affection pulmonaire mal soignée, s'aggravant entre 50 et 60 ans, soit entre 1798 et 1808 , au moment de sa plus grande activité d'homme public, qui ne favorise pas une thérapie tranquille et continue;

- 1808 : prise en charge de la maladie de Ginguené par le médecin des Idéologues qu'est Moreau de la Sarthe qui lui permet quelques rémissions;

- 1815-1816 : phase finale de la tuberculose pulmonaire de l'auteur de l'Histoire littéraire de l'Italie, il dépérit à vue d'œil; ramené d'urgence de Saint-Prix à Paris, Il y meurt en deux jours, les poumons complètement nécrosés par le mal tuberculeux.

Le moins que l'on puisse dire de l'évolution de cette tuberculose pulmonaire, c'est qu'elle fut lente, puisqu'elle a laissé, au dire même de Moreau de la Sarthe, 40 années de rémission à Ginguené.

Quant au rapprochement de celui-ci avec un compatriote qui semble bien être Laennec, il n'engage que Moreau de la Sarthe et sa conception - assez partagée à l'époque - d'une prédisposition des rachitiques à la tuberculose pulmonaire et à... l'intellectualité ! Par une sorte de compensation physiologique, le développement du cerveau se fait au détriment des autres organes; d'où le rachitisme général et la constriction particulière des poumons génératrice de tuberculose.

\section{Index explicatif de certains termes médicaux du texte, peu usuels aujourd 'hui :}

- Affection catarthale : affection due à un écoulement inhabituel, et donc pathologique, d'une muqueuse quelconque; ici, du poumon (catarrhe pulmonaire qui ne se réduit pas toujours, loin de là, au bon gros rhume).

- Disposition érysipélateuse :

inflammation superficielle de la peau avec rougeurs, généralement accompagnée de fièvre; ce peut être le symptôme de beaucoup de choses.

- Hémoptysies:

crachements de sang, plus particulièrement produits par une hémorrhagie de la membrane du poumon; c'est un symptôme très 
explicite de la « phthisie tuberculeuse », c'est-à-dire de la tuberculose pulmonaire

- Mélanose:

développement inhabituel, et donc pathologique (nécrose), de tissus noirs sur différents points du corps; c'est le signe manifeste d'une altération du sang, pouvant elle-même résulter d'une pathologie du poumon.

- CEdématie :

= œdème; l'œdème des membres inférieurs est évidemment le signe d'une mauvaise circulation du sang liée à une déficience organique (du cœur et/ou des poumons) certaine.

- Pectoriloquie :

phénomène de passage des sons de la voix directement du poumon au stéthoscope; c'est évidemment un signe d'existence de cavernes dans les poumons, et donc un symptôme de tuberculose pulmonaire.

- Sueurs colliquatives:

sueurs semblant résulter de la liquéfaction des parties solides du corps; répétées et abondantes, elles épuisent celle ou celui qui en est atteint; elles constituent alors le signe d'une affection grave.

Faculté de Lettres et de Sciences Humaines

Université Paris XII - Val de Mame 